\title{
The 4th Scottish Maths Support Network meeting 2011
}

Shazia Ahmed

Student Learning Service

University of Glasgow

shazia.ahmed@glasgow.ac.uk

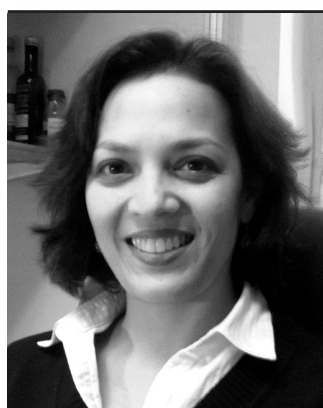

Sue Barnes

Mathematics Skills Support Centre

University of Strathclyde

sue.barnes@strath.ac.uk

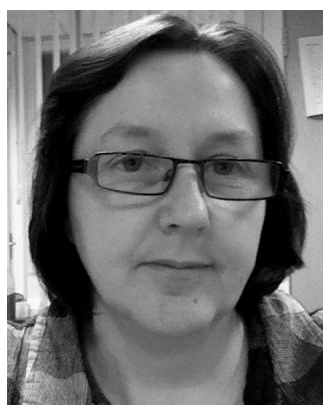

Kate Durkacz

School of Engineering \& Built Environment Edinburgh Napier University

k.durkacz@napier.ac.uk

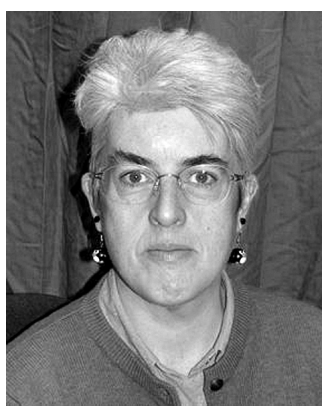

\begin{abstract}
This year the annual meeting of the Scottish Maths Support network (SMSN) was held at the University of Strathclyde on Friday 9 September. Almost all Scottish universities were represented along with one attendee from Ireland. The meeting was organised by the SMSN committee and was sponsored by the MSOR Network. The aim of these annual meetings is to improve awareness of the progress of the Mathematics Support provision at the various institutions, and also to keep up-to-date with current thinking on the running of such support systems.
\end{abstract}

A brief overview of each presentation has been summarised.

\section{We have the technology:}

the role of technology and online resources in Maths \& Stats support

Sue Barnes, University of Strathclyde

While there is an abundance of resources, software packages and technologies available that can be used to provide online support of the learning of Mathematics and Statistics, in many cases we can argue that the most effective support that meets the needs of the individual is one-to-one and face-to-face personal attention.

Support provided as additional online notes or explanation or as online formative assessment has a place in supporting learning and there is a wealth of such resources on the web. In many cases this is all that a student will need. However, no such provision allows the student to ask their own questions and to receive tailored feedback. The process of formulating questions provides an opportunity for analysing understanding that is not provided through prepared content or quizzes. The provision of online discussion, with tutors or peers through forums or messaging does allow for this but as yet there are few easily applied applications that make online discussion of Mathematics accessible. However, such applications are becoming more prevalent and effective.

Technologies, such as the SmartPen, online maths or latex editors, MathType in Office applications, video capture, provide examples. Interactive materials that support active learning provide another way forward. In selecting the help required through interaction with the resource, the user engages with the topic and reviews their understanding.

Thus technologies can contribute to the provision of online Maths \& Stats support, bringing the support experience closer to the one to one and face to face personal support scenario. 


\section{Supporting Students learning in Statistics and Research Methods: ways of coping with a diverse student population}

Jim Kelly, University of Abertay

Quantitative statistics is a subject studied by a diverse population of both undergraduate and postgraduate students at the University of Abertay. These students are studying for qualifications in different subject areas and are often taught statistics using a range of teaching methods. The percentage of statistical content of the various courses also covers a broad range, as does the knowledge of specialised statistical software packages. Thus, providing statistics support for the variety of issues involved can be a challenge.

At the University of Abertay, study skills support is provided, and statistics support is a recent addition to the support resources which has been developed in response to difficulties being experienced by the students. In an effort to encourage students to make use of the statistics support, humorous posters have been developed to advertise the sessions. The support material has been designed to be both relevant and of interest to the students, so for example the mean and standard deviation are explained with reference to the heights of members of boy bands. A new development is the investigation into the use of animations in some of the support material.

\section{Funding Maths support: a consideration of some alternatives}

\section{Katharine Preedy, University of St Andrews}

The structure and staffing of maths support centres varies from university to university and in the current economic circumstances there is increasing pressure on university services to justify their cost. Maths Support at the University of St Andrews has recently operated under a number of different staffing regimes and this has had an effect on the take up provision in the centre, both in terms of numbers of appointments and satisfaction. The different staffing scenarios have included a permanent part time coordinator with tutors on casual contracts; tutors on casual contracts, selected and trained by the coordinator and managed by administrative staff; tutors on casual contracts selected and managed by administrative staff; and a permanent full time coordinator but no tutors. In the absence of permanent staffing provision student engagement with the support provision has fallen significantly which can be attributed to a number of issues including consistency, availability and quality of support. However, it would be reasonable to interpret these findings to support the case for adequate funding and prioritisation of maths support despite the budget constraints that universities are subject to.

\section{Building Sustainability into Formative E-Assessment in Mathematics Support}

\section{Shazia Ahmed, University of Glasgow}

Recent studies have shown that many students lack knowledge of basic mathematical concepts needed at university. Students pursuing scientific courses are often (unpleasantly) surprised to find that their studies include an element of Mathematics. Many of these students have not pursued Mathematics past the age of 16 and find they struggle to perform basic calculations.

As part of the Mathematics Support provision at the University of Glasgow an internal project was funded, aimed at creating a collection of basic questions in Mathematics which can be customised for use in a variety of disciplines. The aim was to enable students to learn mathematics by providing questions for practising mathematical skills. In addition, contextualised questions to suit their discipline were provided to make the learning experience more meaningful and useful.

Moodle quizzes that currently exist for Level 1 Biology students were converted into randomised questions in QTI format. In addition, a 2-session training course was run for staff introducing the MathAssess QTI tools and giving participants hands-on experience of using them. The new quizzes were presented to a group of Biology Level 1 and Summer School students and positive feedback was received. The questions and tests developed in this project will be released as OER under a Creative Commons (CC-BYSA) licence.

\section{The QTI Implementation, Profiling and Support Project}

Sue Milne, ELandWeb Ltd

The QTI-IPS project, funded by JISC, is working to produce a final QTIV2.1 specification and to provide online support for adopters of the spec. Sue Milne talked about the project and how it fits in with Maths Support.

Using standards makes it easier to share resources and to ensure that resources do not become obsolete along with the tools used to create and deliver them. QTIv2.1 already contains the facilities for numerical questions involving mathematical functions, and a suite of Maths extensions for QTIV2.1 have been constructed, enabling authors to write randomised questions which can accept and analyse mathematical input to provide the detailed feedback so important for formative assessment. Questions can be written as context-free maths in the first instance, and various contexts can be added to engage students from different disciplines.

QTI-IPS has collected resources to assist adopters of QTIV2.1 in authoring and converting resources. There are renderers for displaying and responding to questions and tests, conversion applications, editors, example questions and 
links to a variety of support information. Two mailing lists are also available, enabling users to contact one another and elicit advice from experienced practitioners.

Further information can be found on the project website http://qti-ips.niallbarr.me.uk/ and on the project blog http://qtisupport.blogspot.com

\section{To expect defeat is nine-tenths of defeat itself}

\section{James Currell, University of Glasgow}

Thirty or more years of teaching statistics to the unwilling has indicated that the expectations of biologists,

psychologists and medical students in relation to statistics and statistical competence is ambiguous. It is unclear whether students expect to be able to understand the processes involved, or whether they are happy to learn the mechanical steps of the calculation. These days there are a wide variety of computer packages which handle statistical calculations, but maybe it is better to be able to do the calculations by hand and hence gain a deeper understanding of the subject. For the students concerned, the statistics that they use is only a part of their overall programme of study, and is taught as a service course by the staff involved. This clearly has implications for both the depth of study and also for the provision of statistical support provided.

The next Network meeting will be held on 22 June 2012 at the University of St Andrews. An additional event will be held in February 2012 at the University of Glasgow which will focus on developing QTi compliant questions and resources. More details will be available nearer the time. Please email smsn-enquiries@gla.ac.uk for more information.

\section{sigma Visiting Fellow Vacancy}

Following its recent success as the winner of the Times Higher Award for outstanding Support for Students, sigma's activities within the HE-STEM programme are reaching an exciting climax with the new funded centres call and the Practice Adopters Partnership scheme to enhance mathematics support provision. The sigma Directors are seeking to recruit 1.0 fte Visiting Fellow until 31 July 2012 to assist in bringing sigma's activities to a successful conclusion and in securing a lasting legacy from sigma's work within the HE-STEM programme.

The sigma Directors are keen to encourage high quality candidates to apply for this position and are willing to be extremely flexible in the nature of this employment. To this end, they welcome applications from candidates who wish to engage on a fractional rather than full-time basis (and share the position with others); who may have particular strengths in relation to one (but not necessarily all) of the areas of work.

Applications to undertake this work on a secondment basis (again, possibly part-time) are welcome. The nature of the work to be undertaken does not require a frequent presence in Coventry and the post will be available as 'location independent working'.

For more information go to http://sigma-network.ac.uk or contact DagmarWaller d.a.waller@bham.ac.uk 